\title{
Día de GeoGebra desde una Perspectiva Interdisciplinaria en la Educación
}

\author{
Clara R. Moncada Andino ${ }^{1}$ \\ ${ }^{1}$ Instituto GeoGebra de Zacatepec - Departamento de Ciencias Básicas \\ Tecnológico Nacional de México (TecNM)/Sede Zacatepec \\ Código Postal 62780 - Morelos - México \\ clara.moncadalgmail.com
}

\begin{abstract}
The IGZ's birth is celebrated on December $19^{\text {th }}$ of 2013, date when the application was received by email, even though the application's document was signed on December 4 by Markus Hohenwarter, creator of GeoGebra. The main and most valuable action of the GeoGebra Institute of Zacatepec (IGZ) is its call for GeoGebra Day, which has been celebrate twice per year since 2015, one per each semester academic period. This activity is addressed, exclusively, to students of the different engineering careers of the National Technological Institute of Mexico, Zacatepec headquarters, which makes the event rich an interesting because it is interdisciplinary, with the defense of projects of interest.
\end{abstract}

Resumen. La fecha de nacimiento del IGZ se celebra el 19 de diciembre del 2013, que es cuando fue recibida la aplicación por correo electrónico, aunque el documento de aplicación fue firmado por Markus Hohenwarter, creador de GeoGebra, el cuatro de diciembre. La principal y más valiosa acción del Instituto GeoGebra de Zacatepec (IGZ), es su convocatoria para el Día de GeoGebra, que desde el año 2015 se lleva a cabo dos veces al año, uno por cada período académico semestral. La convocatoria va dirigida exclusivamente a estudiantes de las diferentes carreras de ingeniería del Tecnológico Nacional de México, sede Zacatepec, lo que vuelve al evento rico e interesante por ser interdisciplinario, con la defensa de proyectos de interés.

\section{Generales sobre el Día de GeoGebra}

El Instituto GeoGebra de Zacatepec (IGZ) realiza este evento dos veces al año, uno cada semestres, de momento, dirigido exclusivamente a estudiantes de las carreras de ingeniería química y bioquímica, sistemas computacionales, civil, electromecánica, industrial, gestión empresarial, administración; incluyendo además a las licenciaturas de turismo y administración.

Se comparte este trabajo basado en la experiencia que desde la primera convocatoria se ha realizado consecutivamente hasta ahora cada semestre académico: uno de enero a junio, y el otro de agosto a diciembre de cada año.

El objetivo principal del evento: Día de GeoGebra, es fomentar entre los estudiantes el uso de las tecnologías de la información con fines académicos, específicamente el aprendizaje de Geometría, Álgebra y Cálculo [2015, pág. 24], 
esperando que los resultados logrados mejoren la calidad de su formación integral, así como el uso de GeoGebra en las otras asignaturas que conforman sus planes de estudio. Asimismo, de manera indirecta, establecer puentes de interés en un mayor número del cuerpo docente del plantel.

\section{Convocatoria del Día de GeoGebra}

La intención didáctica de este evento es fortalecer la relación teórico-práctica del conocimiento, principalmente, en este caso, de matemáticas: geometría, álgebra, cálculo, etc.; de tal manera que el estudiante llegue al diseño de situaciones teóricas de matemáticas, como también de cualquier otra asignatura de ciencias básicas.

GeoGebra es un recursos tecnológico que se adecúa y cubre algunas de las necesidades de la sociedad del conocimiento para el desarrollo de habilidades como el arte de pensar e impulsar el razonamiento abstracto, el trabajo colaborativo, al considerar escenarios de aprendizaje en equipos y cooperar para aprender; sea que se incluya o no como parte del proceso de evaluación.

Este recurso tecnológico es un excelente punto de partida para desarrolla en los estudiantes las competencias del siglo XXI, y el Día de GeoGebra es una estrategia que permite a los estudiantes desarrollar cómo descubrir algunas de sus potencialidades de formación en el conocimiento matemático e integral de su perfil profesional. Gracias a ello, los docentes de los estudiantes que participan en este evento, se ven influenciados por la original creatividad del estudiante, en cuanto al conocimiento que el docente enseña y el estudiante logra extrapolar e implementar.

De esta manera, los docentes que imparten estas materias, valoren e incorporen el uso de GeoGebra como recurso en la planeación y gestión de sus clases

La convocatoria describe el contexto en el cual se lleva a cabo el evento, la fecha del Día de GeoGebra, cómo participar, el formato de entrega, fecha y hora de inscripción, los criterios de valoración, cuándo se anunciarán los resultados, cómo se procederá al respecto y un apartado donde se hace mención de que los premios a los primeros lugares son sorpresa, además de que todos, por el hecho de participar, recibirán una constancia y reconocimiento adicional a los primeros tres lugares.

En el horario del día único de registro, los estudiantes llenan una ficha donde reportan el título de su proyecto, la carrera que estudian, nombres completos, correo electrónico y número de móvil. Asimismo, dejan en electrónico tres archivos: un documento Word donde se desarrolla el proyecto en su totalidad, incluyendo imágenes de la visualización de su trabajo en GeoGebra, otro archivo ggb de la aplicación dinámica y un tercer archivo en PowerPoint, que será con el que defenderán su proyecto el Día de GeoGebra.

Adicionalmente a la convocatoria, se hace difusión del evento en las redes sociales, con el documento de ésta y usando videos; también se entregan trípticos informativos que son entregados en los salones de clase a través de otros estudiantes. El evento comenzó a publicarse en el calendario escolar oficial de la sede de Zacatepec desde el primer período semestral del 2015. La siguiente figura muestra el calendario académico escolar del primer y segundo período semestral de los años 2015 y 2018 respectivamente, donde se incluye el Día de GeoGebra en los meses de abril y octubre. 
VIII Congresso Brasileiro de Informática na Educação (CBIE 2019)

Anais dos Workshops do VIII Congresso Brasileiro de Informática na Educação (WCBIE 2019)
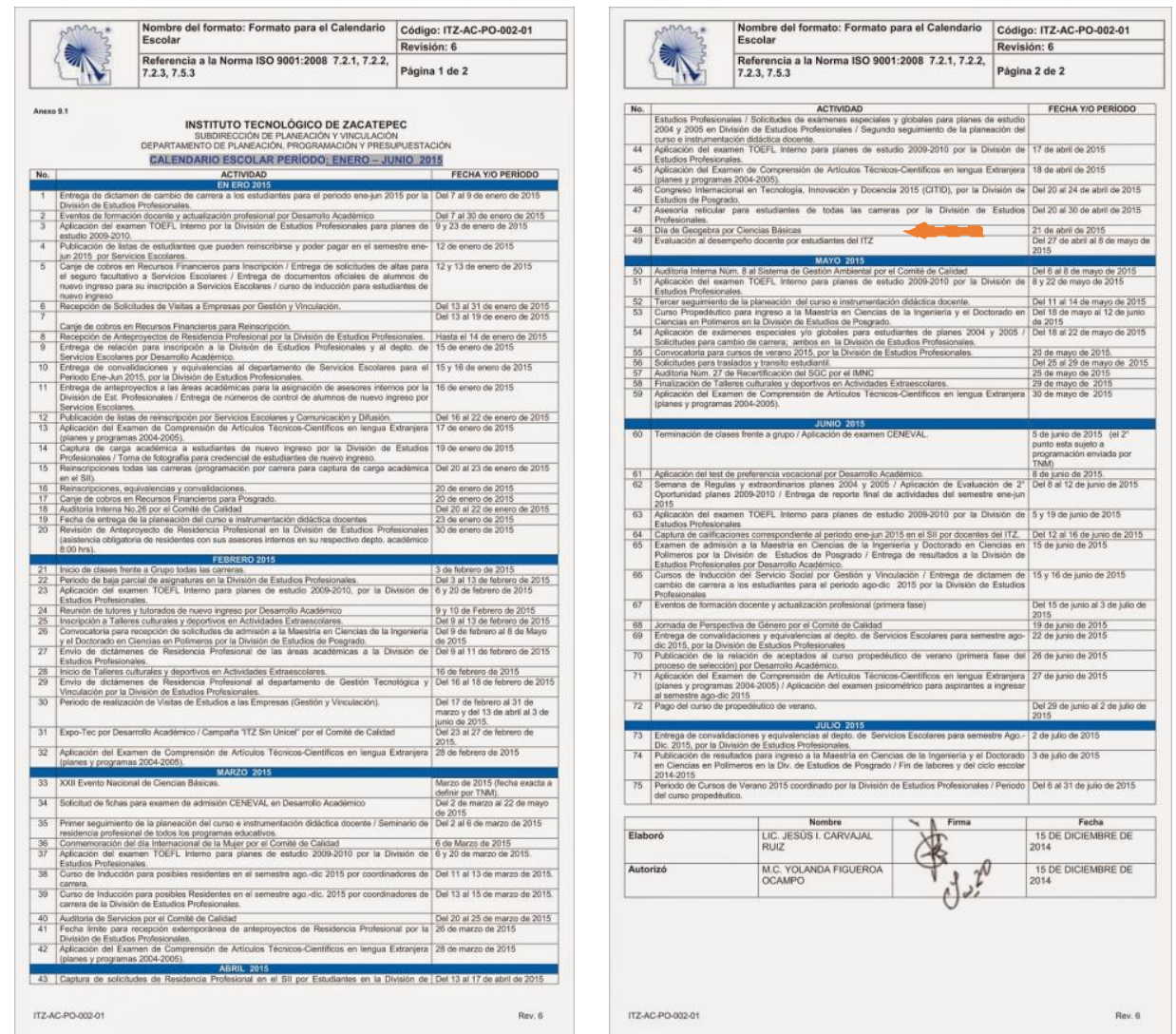

Figura 1. Calendario escolar semestral oficial enero-junio, 2015 [TecNM, 2019].

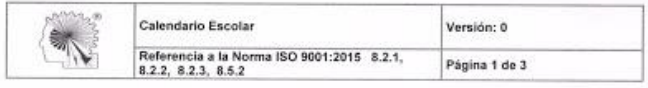

INSTITUTO TECNOLÓGICO DE ZACATEPEC SUBDIRECCION DE PLANEACION Y VNAULLACION
DEPARTAMENTO DE PLANEACION, PROGRAMACIÓN Y PRESUPUESTACION

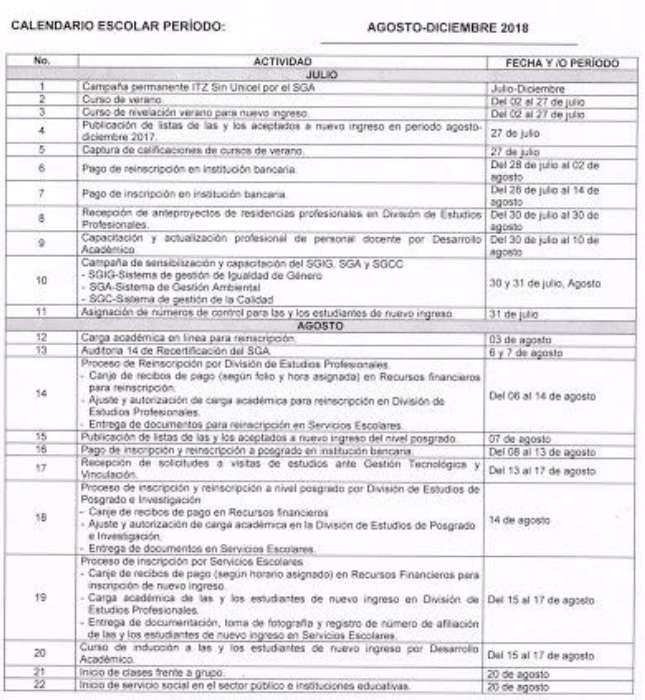

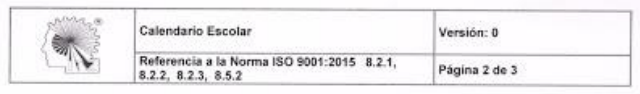

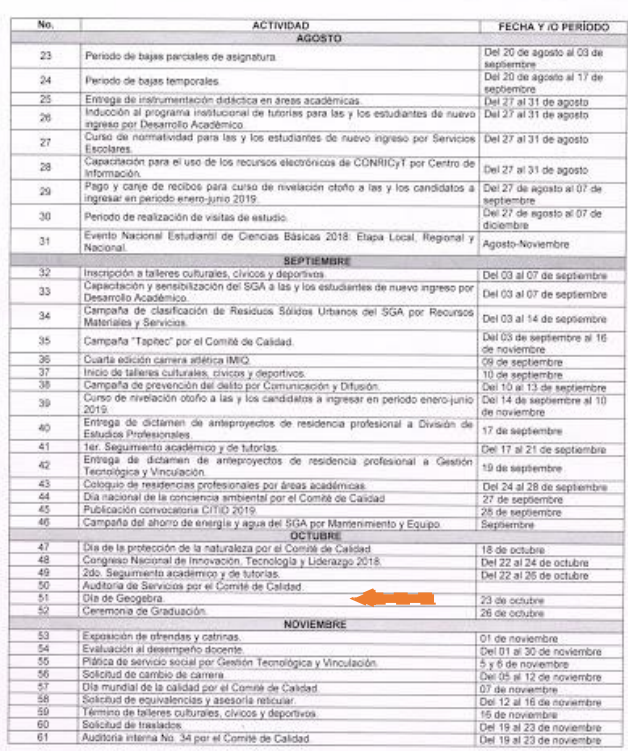

Figura 2. Calendario escolar semestral oficial agosto-diciembre, 2018 [TecNM, 2019]. 
VIII Congresso Brasileiro de Informática na Educação (CBIE 2019)

Anais dos Workshops do VIII Congresso Brasileiro de Informática na Educação (WCBIE 2019)

Las siguientes figuras son de las convocatorias correspondientes a los períodos antes descritos en los calendarios.
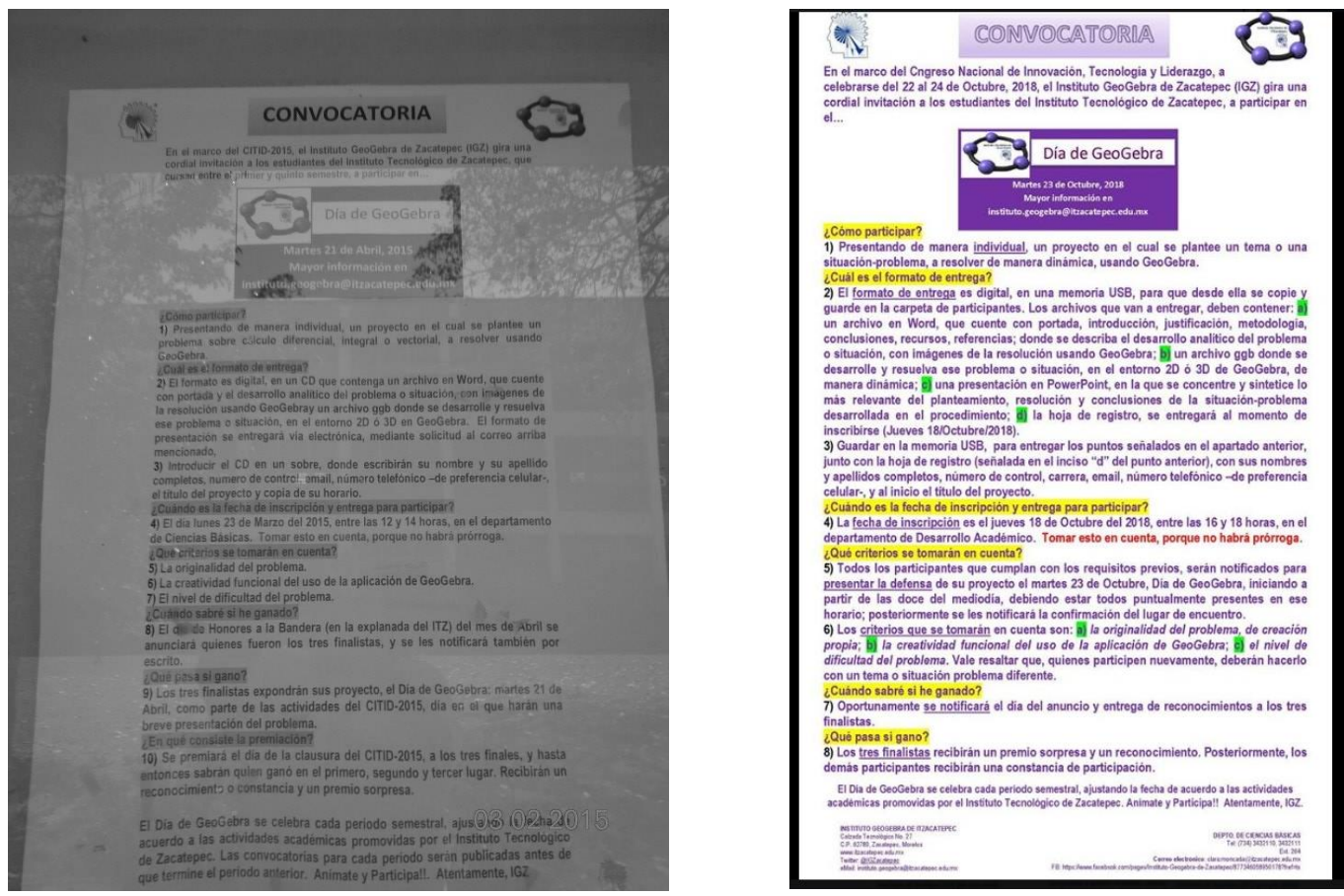

Figura 3. Convocatorias asociadas a los calendarios de las figuras 1 y 2.

\section{Proceso de Revisión de Proyectos}

Los archivos de los estudiantes registrados se envían por correo a los jurados, que han sido previamente invitados, junto con una rúbrica donde se plasman los criterios de valoración, de esta manera, el Día de GeoGebra, en el que los proyectos son defendidos, cada jurado termina de realizar la valoración individual.

Concluidas las defensas, los jurados se reúnen para decidir a los ganadores, tomando en cuenta el puntaje que le hayan dado a cada participante, y de esta concentración de resultados se deciden los primeros tres lugares.

Los criterios de valoración se fundamenta en la originalidad del tema del proyecto presentado, la creatividad de la aplicación dinámica en GeoGebra y el grado o nivel de dificultad; asimismo son consideradas la seguridad al momento de defender el proyecto usando la presentación y dar respuestas sustentadas a las preguntas hechas por cada uno de los jurados, después de haber disertado.

\subsection{Algunos Temas Ganadores Defendidos}

Hasta ahora los temas presentados se han centrado exclusivamente en matemáticas. La siguiente es una relación de algunos de los temas presentados en los proyectos:

Área entre curvas con incidencia.

Área bajo una curva.

Optimizando con funciones lineales.

Aplicaciones en programación lineal. 
VIII Congresso Brasileiro de Informática na Educação (CBIE 2019)

Anais dos Workshops do VIII Congresso Brasileiro de Informática na Educação (WCBIE 2019)

Calcular la presión de una superficie plana con cálculo integral.

Pitágoras.

Funciones reales de varias variables.

Fuerzas con vectores.

Calcular la distancia en una variable.

Volumen de un contenedor de agua.

La derivada.

Aplicaciones de la derivada.

En la figura se visualiza un ejemplo de carpeta conteniendo los archivos de un participante registrado.

\begin{tabular}{|c|c|c|c|c|}
\hline \multicolumn{5}{|c|}{ - Memoria_ProyectosRegistrados_Dia_GeoGebra >02_OrtizParraReyDavid } \\
\hline \multirow[t]{3}{*}{$\operatorname{lod}^{\wedge} \wedge$} & Nombre & Fecha de modifica... & Tipo & Tamaño \\
\hline & PLITAG PITAGORAS POWER & 24/03/2017 16:52 & Microsoft PowerPo... & $513 \mathrm{~KB}$ \\
\hline & w PITAGORAS & 24/03/2017 16:51 & Microsoft Word D... & $578 \mathrm{~KB}$ \\
\hline $19-Y$ & PITAGORAS & 22/03/2017 23:04 & GeoGebra file & $18 \mathrm{~KB}$ \\
\hline
\end{tabular}

Figura 4. Carpeta del participante Rey David Ortiz Parra de la carrera de ingeniería electromecánica, siendo el tema de su proyecto Pitágoras.

\subsection{De los Estudiantes que han Participado}

En primer lugar, el interés se manifiesta porque es un evento libre de cobro; por otro lado, ninguno de los estudiantes participantes ha contado con asesor, ellos investigan por sí mismos y deciden el proyecto; en ocasiones su participación está relacionada con alguna de las asignaturas que cursan.

Los participantes han sido voluntarias con estudiantes de las carreras de ingeniería civil, electromecánica, industrial y sistemas computacionales.

Las figuras de a continuación se muestran algunas fotografías de diferentes participaciones y entrega de constancias de Días de GeoGebra.
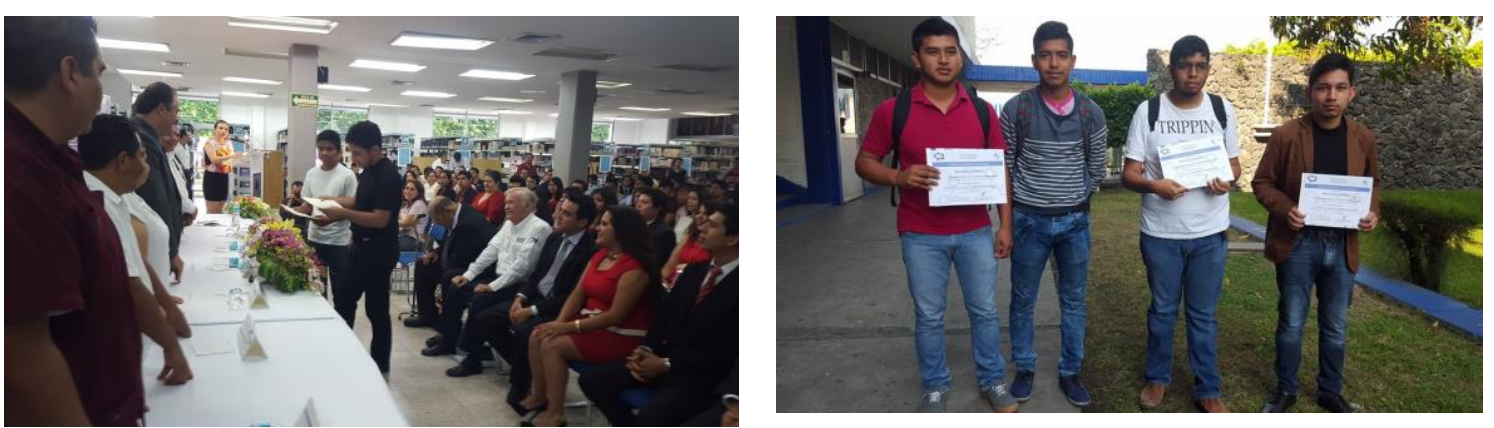

Figura 5. En la foto de la izquierda reciben reconocimientos y constancias en la clausura del contexto académico llevado a cabo, en el cual el Día de GeoGebra se incluye. En la derecha los tres primeros lugares de otro Día de GeoGebra, el de camisa roja es Rey David, de quien se muestra la carpeta en la figura 4. 
VIII Congresso Brasileiro de Informática na Educação (CBIE 2019)

Anais dos Workshops do VIII Congresso Brasileiro de Informática na Educação (WCBIE 2019)
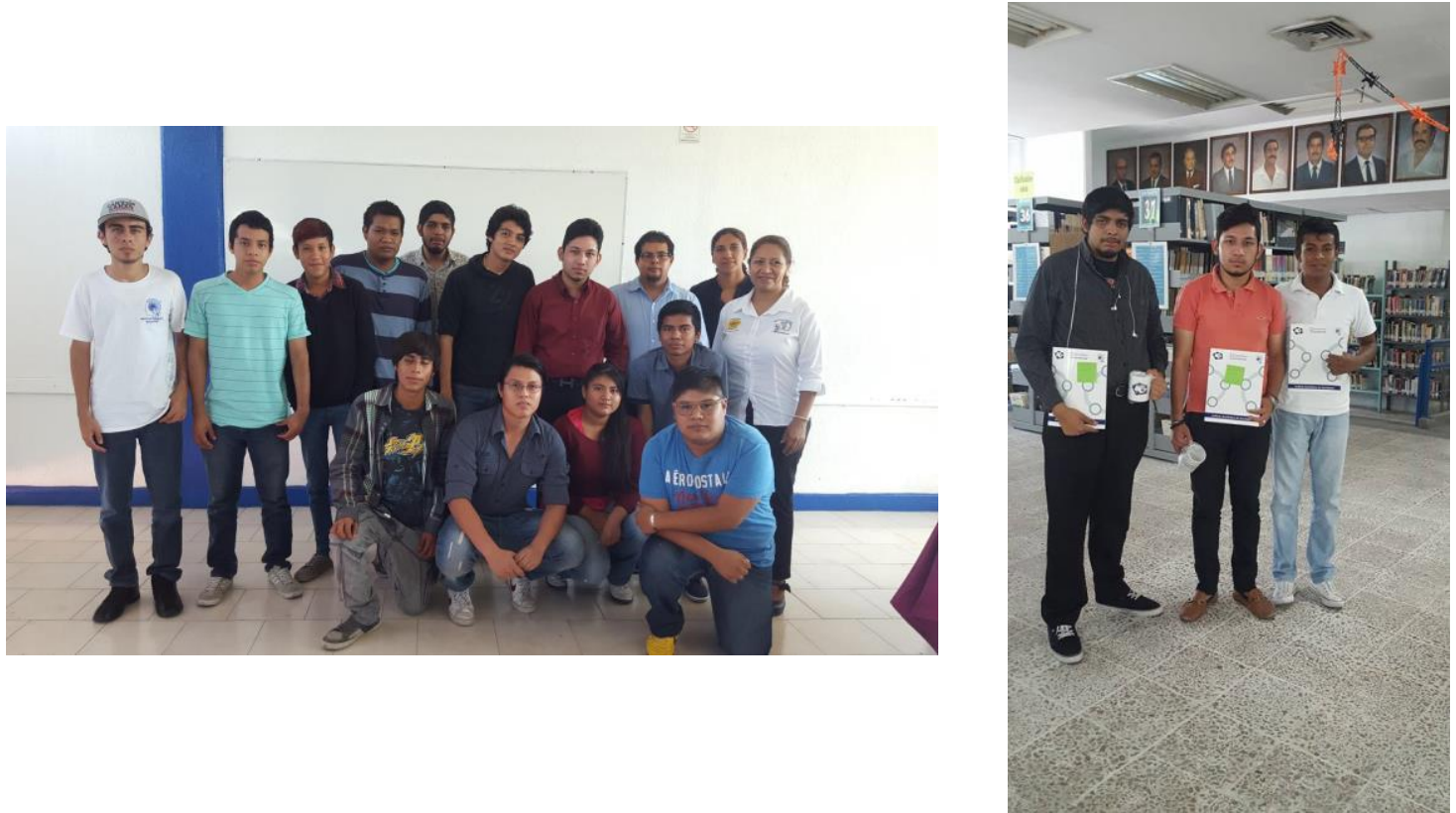

Figura 6. La foto de la izquierda muestra un grupo de participantes y tres de los cuatro jurados. En la foto de la derecha están tres ganadores de los primeros tres lugares en otro Día de GeoGebra, quienes participaron dos y tres veces diferentes y ahora hacen residencia como paso previo a graduarse de ingenieros, son de las carreras de ingeniería en sistemas computaciones e industrial el de la derecha.

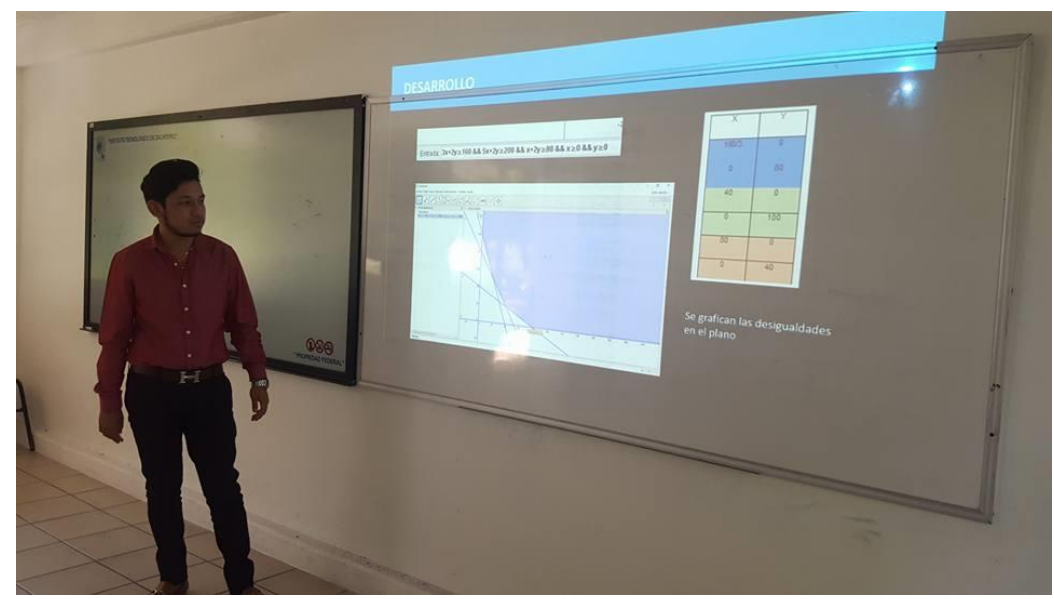

Figura 7. Defendiendo uno de los proyectos de las tres participaciones, mismas que consecutivamente ganó.

\section{3. Áreas Departamentales de los Jurados}

Los jurados son docentes de la sede de Zacatepec, que ha tomado algún curso de GeoGebra y que parcialmente lo utilizan como recurso. No son siempre los mismos y están adscritos al departamento de ciencias básicas y a las carreras de sistemas computacionales, civil, electromecánica, química y bioquímica. 
VIII Congresso Brasileiro de Informática na Educação (CBIE 2019)

Anais dos Workshops do VIII Congresso Brasileiro de Informática na Educação (WCBIE 2019)

Ellos reciben una constancia por su participación como jurado, fungiendo como tales de manera muy profesional.

Como convocante y autora de este trabajo, el rol de jurado queda excluido, ya que no se puede ser juez y parte del evento, además de que es frecuente que estudiantes propios participan.

\section{Resultados}

A la fecha se han registrado un total de cincuenta y siete estudiantes, incluyendo a los de este período agosto-diciembre. El número de inscritos varía de semestre a semestre y en este último los participantes son de las carreras de ingeniería civil, industrial y sistemas computacionales. Esta interdisciplinaridad favorece a estudiantes por la oportunidad que este escenario les brinda para empoderarse del conocimiento de manera dinámica al utilizar GeoGebra. A través del estudiante participante se favorece también el docente, al evidenciar el potente recurso tecnológico que este software es.

Los temas de los proyectos registrados en el período agosto-diciembre del 2019, abordan los siguientes contenidos: probabilidad, cálculo vectorial, astronomía, geometría, esferas en una figura octogonal, funciones relacionadas a un cuadrilátero, representación de la doble hélice de ADN y tiro parabólico.

El Día de GeoGebra se ha oficializado en el calendario académico escolar, ha sido posible la divulgación del evento, sin embargo, la inigualable ayuda de difusión son los mismos estudiantes que se han animado a participar e invitan a otros a que lo hagan al compartir con ellos la experiencia vivida, el beneficio que en cuanto a conocimientos del contenido que conlleva el proyecto han logrado dominar y mejorar el uso constructivo y significativo de y con GeoGebra.

Otro resultado de interés y beneficio estudiantil es el premio que además del reconocimiento y constancia reciben: los premios sorpresas, varían en porcentajes de descuento en la reinscripción al siguiente semestre, libros y dinero, todos ellos en proporción a los tres primeros lugares en el que hayan quedado.

En los primeros semestres de los años 2015, 2017 y 2019 en contexto académico del Día de GeoGebra fue parte del Congreso Internacional de Tecnología, Innovación y Docencia (CITID) que se lleva a cabo en la sede de Zacatepec.

\section{Conclusiones}

Estudiantes, docentes e institución se benefician con este evento: Día de GeoGebra. Los primeros porque, sin contar con asesor, se registran respetando los señalamientos de la convocatoria, y con ello, fortalecen sus habilidades, conocimientos, competencias integrales de formación profesional y humana.

El beneficio del docente surge a través del estudiante que le comparte, sea que haya o no ganado uno de los primeros tres lugares, el proyecto con el cual participó, y es entonces que el docente, en algún momento, podría interesarse a pesar de la resistencia que el uso de las tecnologías de la información implica en él. El estudiante es clave para que el docente voluntariamente se acerque a GeoGebra como recurso para la enseñanza. 
VIII Congresso Brasileiro de Informática na Educação (CBIE 2019)

Anais dos Workshops do VIII Congresso Brasileiro de Informática na Educação (WCBIE 2019)

Desde el momento en el que la institución incluye en el calendario escolar, rendición de cuentas y congresos, es porque recibe un beneficio para la mejora de la calidad educativa. Antes de que cierre cada período académico semestral y se inicia con la programación del siguiente calendario escolar, la institución informa sobre la fecha en la que se realizarán eventos académicos para definir e incluir el Día de GeoGebra.

Mientras tanto GeoGebra sigue mejorando su interfaz continuamente, manteniendo con ello la puerta abierta a las diversas áreas de oportunidades que se pudieran presentar, tanto su uso en las matemáticas, como en las otras ciencias, en cualquier nivel educativo o rubro profesional fundamentalmente en la formación de profesores de matemáticas.

\section{Referencias}

Moncada, C. (2019). Experiencias interdisciplinarias del evento Día de GeoGebra no documentada. Instituto GeoGebra de Zacatepec. Tecnológico Nacional de México (TecNM)/Sede Zacatepec. México.

Tecnológico Nacional de México (TecNM)/sede Zacatepec. (2015). Informe de rendición de cuentas 2015 del Instituto Tecnológico de Zacatepec. Tomado el 20 de octubre del 2019 de https://studylib.es/doc/7394847/sin-t\%C3\%ADtulo-1---institutotecnologico-de-zacatepec. Páginas 15, 17, 24, 44. México.

Tecnológico Nacional de México (TecNM)/sede Zacatepec. (2019). Blog del Instituto Tecnológico de Zacatepec: Calendario semestral escolar oficial. Tomado el 21 de septiembre del 2019 de https://itdezacatepec.blogspot.com/?fbclid=IwAR3NRnKYEYZhhtAT6VgWO3O9ir ru13_zNgQxcbnuut85qu iTmkvveIvhI. 